\title{
On the Torsion Subgroups of Certain Elliptic Curves over $\mathbb{Q}^{*}$
}

\author{
Yoon Kyung Park \\ School of Mathematics, Korea Institute for Advanced Study, Seoul, Republic of Korea \\ Email: ykpark@math.kaist.ac.kr
}

Received October 2, 2012; revised December 3, 2012; accepted December 15, 2012

\begin{abstract}
Let $E$ be an elliptic curve over a given number field $K$. By Mordell's Theorem, the torsion subgroup of $E$ defined over $\mathbb{Q}$ is a finite group. Using Lutz-Nagell Theorem, we explicitly calculate the torsion subgroup $E(\mathbb{Q})_{\text {tors }}$ for certain elliptic curves depending on their coefficients.
\end{abstract}

Keywords: Elliptic Curve; Rational Point

\section{Introduction}

A cubic curve over the field $K$ in Weierstrass form is given by projectively

$$
y^{2} w+a_{1} x y w+a_{3} y w^{2}=x^{3}+a_{2} x^{2} w+a_{4} x w^{2}+a_{6} w^{3},
$$

with coefficients in $K$. Then there is a unique $\bar{K}$ rational point $(x, y, w)=(0,1,0)$ on the line at infinite $w=0$. If the above is an elliptic curve, then $(0,1,0)$ is a nonsingular point and we deal with the curve by working with the affine form

$$
y^{2}+a_{1} x y+a_{3} y=x^{3}+a_{2} x^{2}+a_{4} x+a_{6} .
$$

Hereafter assume that $K$ is a number field. Since the field characteristic of $K$ is 0 , we can study

$$
y^{2}=x^{3}+A x+B
$$

instead of (1.1). When the discriminant $\Delta_{E}=4 A^{3}-27 B^{2}$ is nonzero, $E$ is a nonsingular curve. By Mordell's theorem, $E(K)$ is a finitely generated abelian group and its torsion subgroup $E(K)_{\text {tors }}$ is a finite abelian group. Mazur proved that $E(\mathbb{Q})$ of an elliptic curve $E$ over the rational numbers must be isomorphic to one of the following 15 types [1]:

$$
\begin{aligned}
& \mathbb{Z} / N \mathbb{Z}, N=1-10,12 \\
& \mathbb{Z} / 2 \mathbb{Z} \oplus \mathbb{Z} / 2 N^{\prime} \mathbb{Z}, N^{\prime}=1-4 .
\end{aligned}
$$

This paper is focused on knowing how the coefficients $A$ and $B$ of $(1.2)$ determine $E(\mathbb{Q})_{\text {tors }}$. For the earlier work, we see the cases $A$ or $B$ is zero in [2]:

Theorem 1. Let $E$ be the elliptic curve $y^{2}=x^{3}+A x+B$ with $A$ and $B$ in $\mathbb{Z}$.

\footnotetext{
"This work was supported by NRF 2012-0006901.
}

1) If $A$ is fourth-power free and $B=0$, then

$$
E(\mathbb{Q})_{\text {tors }}= \begin{cases}\mathbb{Z} / 2 \mathbb{Z} \oplus \mathbb{Z} / 2 \mathbb{Z}, & \text { if }-A \text { is a square in } \mathbb{Z}, \\ \mathbb{Z} / 4 \mathbb{Z}, & \text { if } A=4, \\ \mathbb{Z} / 2 \mathbb{Z}, & \text { otherwise. }\end{cases}
$$

2) If $B$ is sixth-power free and $A=0$, then

$$
\begin{aligned}
& E(\mathbb{Q})_{\text {tors }} \\
& =\left\{\begin{array}{l}
\mathbb{Z} / 6 \mathbb{Z}, \text { if } B=1, \\
\mathbb{Z} / 3 \mathbb{Z}, \text { if } B=-432=-2^{4} 3^{3}, \text { or if } B \text { is square not } 1, \\
\mathbb{Z} / 2 \mathbb{Z}, \text { if } B \text { is cubic not } 1, \\
0, \quad \text { otherwise. }
\end{array}\right.
\end{aligned}
$$

It is too hard to determine the group $E(\mathbb{Q})_{\text {tors }}$ without any relation between the coefficients. Hence we consider the elliptic curve as follows:

$$
y^{2}=x^{3}+f(k) x+g(k)
$$

with $f(k), g(k) \in \mathbb{Z}[k]$. Then Theorem 1 yields the case when one of $f(k)$ and $g(k)$ is zero and $\max \left\{\operatorname{deg}_{k} f(k), \operatorname{deg}_{k} g(k)\right\}=1$. In this paper, we deal with the case $\max \left\{\operatorname{deg}_{k} f(k), \operatorname{deg}_{k} g(k)\right\}=2$.

Theorem 2. Let

$$
E: y^{2}=x^{3}-(6 k+3) x-\left(3 k^{2}+6 k+2\right)
$$

be the elliptic curve with $k$ in $\mathbb{Z}$. Suppose that $k$ is an integer such that $35 \nmid k(9 k+4)$ and there is no integer $h$ satisfying $k=4 h\left(3 h^{2}+3 h+1\right)$ or $-4(h+1)\left(3 h^{2}+3 h+1\right)$. Then 


$$
E(\mathbb{Q})_{\text {tors }}= \begin{cases}\mathbb{Z} / 4 \mathbb{Z}, & k \equiv 20 \text { or } 34(\bmod 35), \exists l \in \mathbb{Z} \text { such that } k=-3 l^{2}(1+l) \text { and } \\ \exists m \in \mathbb{Z} \text { satisfying } m^{2}=l(3 l-2) \text { and } 6\left(6 l^{2}-5 l m-2\right) \text { is square, } \\ \mathbb{Z} / 2 \mathbb{Z}, & k \equiv 20 \text { or } 34(\bmod 35), \exists l \in \mathbb{Z} \text { such that } k=-3 l^{2}(1+l) \text { and } \\ \nexists m \in \mathbb{Z} \text { satisfying } m^{2}=l(3 l-2) \text { and } 6\left(6 l^{2}-5 l m-2\right) \text { is square, } \\ \mathbb{Z} / 2 \mathbb{Z}, & k \text { is congruent to one of the elements of the set } K_{2} \text { modulo } 35 \\ & \text { and } \exists l \in \mathbb{Z} \text { such that } k=-3 l^{2}(1+l), \\ 0, & \text { otherwise. }\end{cases}
$$

where $K_{2}=\{x \in \mathbb{Z} / 35 \mathbb{Z}: x \equiv 4,7,12,15,22,25,27,29,32\}$.

\section{The Proof of Theorem 2}

We use the Lutz-Nagell Theorem and we have to calculate $E_{p}\left(\mathbb{F}_{p}\right)$ if $E$ has a good reduction at the prime $p$.

Theorem 3. (Lutz-Nagell) Let $E$ be an elliptic curve (1.1) with coefficients in $\mathbb{Z}$ and $E_{p}$ be a obtained curve by reducing coefficients of $E$ modulo $p$. And let $\Delta_{E}$ be the discriminant of $E$.

1) If $a_{1}=0$ and if $P=(x(P), y(P), 1)$ is in $E(\mathbb{Q})_{\text {tors }}$, then $x(P)$ and $y(P)$ are integers;

2) For any $a_{1}$, if $P=(x(P), y(P), 1)$ is in $E(\mathbb{Q})_{\text {tors }}$, then $4 x(P)$ and $8 y(P)$ are integers;

3) If $p$ is an odd prime such that $p \nmid \Delta_{E}$, then the restriction to $E(\mathbb{Q})_{\text {tors }}$ of the reduction homomorphism $r_{p}: E(\mathbb{Q}) \rightarrow E_{p}\left(\mathbb{Q}_{p}\right)^{\text {trs }}$ is one-to-one. The same conclusion is valid for $p=2$ if $2 \nmid \Delta_{E}$ and $a_{1}=0$;

4) If $a_{1}=a_{3}=a_{2}=0$, so that $E$ is given by

$$
y^{2}=x^{3}+A x+B
$$

and if $P(x(P), y(P), 1)$ is in $E(\mathbb{Q})_{\text {tors }}$, then either $y(P)=0$ (and $P$ has order 2) or else $y(P) \neq 0$ and $y(P)^{2}$ divides $d=-4 A^{3}-27 B^{2}$.

Proof. See [2].

Lemma 4. Let $E: y^{2}=x^{3}+A x+B$ be the elliptic curve over $\mathbb{F}_{p}$ and $P=(x, y)$ be a point in $E\left(\mathbb{F}_{p}\right)$ which is not a point at infinity. Then the followings are equivalent.

1) $P=(x, y)$ is a point of order 3 in $E\left(\mathbb{F}_{p}\right)$;

2) $3 x^{4}+6 A x^{2}+12 B x-A^{2}$ is congruent to 0 modulo $p$.

Proof. 1) $\Rightarrow$ 2) Let $\left(x_{2}, y_{2}\right)$ be the point $2 P=P+P$. Then by the group law algorithm ([2]),

$$
\begin{aligned}
x_{2}= & \frac{x^{4}-2 A x^{2}-8 B x+A^{2}}{4 y^{2}} \\
y_{2} & =\frac{-\left(3 x^{2}+A\right)\left(\frac{\left(3 x^{2}+A\right)^{2}}{4 y^{2}}-2 x\right)}{2 y}-\frac{-x^{3}+A x+2 B}{2 y}
\end{aligned}
$$

and

$$
-P=(x,-y) .
$$

Then $3 P=O$ means that

$$
\begin{gathered}
x^{4}-2 A x^{2}-8 B x+A^{2}=4 x y^{2} \\
-\left(3 x^{2}+A\right)\left(\frac{\left(3 x^{2}+A\right)^{2}}{4 y^{2}}-2 x\right)-\left(-x^{3}+A x+2 B\right)=-2 y^{2} .
\end{gathered}
$$

Since $y^{2}=x^{3}+A x+B, \quad x$ should satisfy that $3 x^{4}+6 A x^{2}+12 B x-A^{2}=0$ in $\mathbb{F}_{p}$.

2) $\Rightarrow 1$ ) Assume that $3 x^{4}+6 A x^{2}+12 B x-A^{2}=0$, $y$ is not zero and $y^{2}=x^{3}+A x+B$ in $\mathbb{F}_{p}$. By simple calculation, such $x, y$ satisfy (5) and (6) and if $P$ is the point $(x, y)$ then $2 P=-P$. We are done. $\square$

Here we choose two rational primes 5,7 and calculate the groups $E\left(\mathbb{F}_{5}\right)$ and $E\left(\mathbb{F}_{7}\right)$. For the integer $k$ unmentioned in our main theorem, we can take another prime and apply it as same manner.

Lemma 5. Let $p$ be the rational prime and $E$ be the elliptic curve defined as

$$
y^{2}=x^{3}-(6 k+3) x-\left(3 k^{2}+6 k+2\right)
$$

where $k$ is a nonzero integer. And using the natural surjection from $\mathbb{Z}$ to $\mathbb{F}_{p} \cong \mathbb{Z} / p \mathbb{Z}$, we can get $E_{p}$ by reducing the coefficients of $E$ modulo $p$. If $p$ does not divide the discriminant $-2^{4} \times 3^{3} \times k^{3}(9 k+4)$ then the group $E_{p}$ consisting of the points defined over the finite field $\mathbb{F}_{p}$ with $p$ elements is
1) $E_{5}\left(\mathbb{F}_{5}\right)=\left\{\begin{array}{l}\mathbb{Z} / 9 \mathbb{Z}, k \equiv 1(\bmod 5), \\ \mathbb{Z} / 6 \mathbb{Z}, k \equiv 2(\bmod 5), \\ \mathbb{Z} / 3 \mathbb{Z}, k \equiv 3(\bmod 5) .\end{array}\right.$
2) $E_{7}\left(\mathbb{F}_{7}\right)= \begin{cases}\mathbb{Z} / 3 \mathbb{Z} \oplus \mathbb{Z} / 3 \mathbb{Z}, k \equiv 3(\bmod 7), \\ \mathbb{Z} / 6 \mathbb{Z}, & k \equiv 1,4(\bmod 7), \\ \mathbb{Z} / 9 \mathbb{Z}, & k \equiv 2(\bmod 7), \\ \mathbb{Z} / 12 \mathbb{Z}, & k \equiv 6(\bmod 7) .\end{cases}$ 
Table 1. Point in $\mathbf{E}_{5}\left(\mathbb{Z}_{5}\right)$.

\begin{tabular}{cccc}
\hline$k(\bmod 5)$ & $E_{5}\left(\mathbb{Z}_{5}\right)-\{O\}$ & $\mid E_{5}\left(\mathbb{Z}_{5}\right)$ & generators in $E_{5}\left(\mathbb{Z}_{5}\right)$ \\
\hline 1 & $0,(0, \pm 2),(1, \pm 1),(2, \pm 2),(3, \pm 2)$ & 9 & $0,(0, \pm 2),(1, \pm 1),(2, \pm 2)$ \\
2 & $0,(0, \pm 2),(1,0),(3, \pm 1)$ & 6 & $(3, \pm 1)$ \\
3 & $(2, \pm 2)$ & 3 & $(2, \pm 2)$ \\
\hline
\end{tabular}

Proof. By [3], every $E_{p}\left(\mathbb{F}_{p}\right)$ has a subgroup of $\mathbb{Z} / 3 \mathbb{Z}$. Table 1 is the proof of (1).

Both cases can be calculated as using simple calculation. For 2), since $p=7$ and $p \nmid k(9 k+4), k$ can not be congruent to 0 and $5(\bmod 7)$. When

$k \equiv 1(\bmod 7), E_{7}$ becomes $y^{2}=x^{3}-2 x+3$. By substituting all elements of $\mathbb{F}_{7}$ to $x$ in $E_{7}$, we can find that $E_{7}\left(\mathbb{F}_{7}\right)=\{(1, \pm 3),(2,0),(6, \pm 2), \infty\}$. Since it is an abelian group with 6 elements, $E_{7}\left(\mathbb{F}_{7}\right) \cong \mathbb{Z} / 6 \mathbb{Z}$. Like this, if $k \equiv 4(\bmod 7)$,

$E_{7}\left(\mathbb{F}_{7}\right)=\{(4, \pm 1),(5,0),(6, \pm 1), \infty\} \quad$ has 6 elements. Hence it is isomorphic to $\mathbb{Z} / 6 \mathbb{Z}$.

In the case $k \equiv 2(\bmod 7) \quad E_{7}: y^{2}=x^{3}-x+2$ has a torsion subgroup $\{(1, \pm 3),(2, \pm 1),(6, \pm 3),(0, \pm 3), \infty\}$ over $\mathbb{F}_{7}$. To find the point of order 3 in the elliptic curve as the form ((2) in Section 1), we have to get the root of the equation $3 x^{4}+6 A x^{2}+12 B x-A^{2}=0$ in given field and it is the $x$-coordinate of the order 3 point by Lemma 4. In this case, the equation is

$3(x+1)\left(x^{3}+6 x^{2}+6 x+2\right)$ in $\mathbb{F}_{7}$. Hence there is no point of order 3 except $(6, \pm 3)$ and $E_{7}\left(\mathbb{F}_{7}\right) \cong \mathbb{Z} / 9 \mathbb{Z}$.

For $k \equiv 3(\bmod 7), E_{7}\left(\mathbb{F}_{7}\right)$ has 9 elements. But the equation giving criterion of order 3 is

$3 x(x+1)(x+2)(x+4)$ in $\mathbb{F}_{7}$ and

$(0, \pm 3),(3, \pm 1),(5, \pm 1),(6, \pm 1) \in E_{7}\left(\mathbb{F}_{7}\right)$. Therefore,

$E_{7}\left(\mathbb{Z}_{7}\right) \cong \mathbb{Z} / 3 \mathbb{Z} \oplus \mathbb{Z} / 3 \mathbb{Z}$.

Last, if $k \equiv 6(\bmod 7)$,

$$
\begin{aligned}
& E_{7}\left(\mathbb{F}_{7}\right) \\
& =\{(0, \pm 1),(2, \pm 1),(3, \pm 3),(4,0),(5, \pm 1),(6, \pm 2), \infty\}
\end{aligned}
$$

has only one point $(4,0)$ of order 2 . It means that $E_{7}\left(\mathbb{F}_{7}\right) \cong \mathbb{Z} / 12 \mathbb{Z}$.

To get 1), we use the same process as 2), I omit it. $\square$

Propositions 6 and 7 give the necessary and sufficient condition to have order 2 and 3 points.

\section{Proposition 6. Let}

$E: y^{2}=x^{3}-(6 k+3) x-\left(3 k^{2}+6 k+2\right)$ be the elliptic curve with $k$ in $\mathbb{Z}$. There is a point of order 2 if and only if $k$ is an integer of the form $-3 l^{2}(1-l)$. Moreover, the point of order 2 is unique.

Proof. Assume that $k$ is an integer of the form $-3 l^{2}(1-l)$. Through easy calculation, $k$ satisfies

$k^{2}+6 l^{2} k+9 l^{4}-9 l^{6}=0$. Then $x=3 l^{2}-1$ is a root of $x^{3}-(6 k+3) x-\left(3 k^{2}+6 k+2\right)=0$ and $\left(3 l^{2}-1,0\right)$ is the point of order 2 in $E(\mathbb{Q})$.

Conversely, suppose that the equation of $x^{3}-(6 k+3) x-\left(3 k^{2}+6 k+2\right)=0$ has a solution in $\mathbb{Z}$. To have solution of the equation with respect to $k, x$ should be congruent to 2 modulo 3 . By substituting $3 m-1$ to $x$, the equation becomes $-3\left\{k^{2}+6 k m-9 m^{2}(m-1)\right\}$. Since it has an integral solution, $m=l^{2}$ and $k=-3 l^{2}(1-l)$ for an integer $l$.

Now we show that there is no point of order 2 except $\left(3 l^{2}-1,0\right)$ in $E(\mathbb{Q})$. Assume that $\left(3 l^{2}-1,0\right) \in E(\mathbb{Q})$. Then $k=-3 l^{2}(1-l)$.

$$
\begin{aligned}
& x^{3}-(6 k+3) x-\left(3 k^{2}+6 k+2\right) \\
& =\left(x-3 l^{2}+1\right)\left(x^{2}-\left(1-3 l^{2}\right) x+\left(9 l^{4}-18 l^{3}+12 l^{2}-2\right)\right) .
\end{aligned}
$$

Let $Q(x)$ be $x^{2}-\left(1-3 l^{2}\right) x+\left(9 l^{4}-18 l^{3}+12 l^{2}-2\right)$ with discriminant $-9(3 l-1)(l+1)^{3}$. If the solution of $Q(x)$ exists, then $-(3 l+1)(l-1) \geq 0$. It gives us the value $l=0$ or 1 . Hence $k=0$ and $E$ is singular. $\square$

Proposition 7. Let

$E: y^{2}=x^{3}-(6 k+3) x-\left(3 k^{2}+6 k+2\right)$ be the elliptic curve with $k$ in $\mathbb{Z}$. Assume that there is no integer $h$ such that $k=4 h\left(3 h^{2}+3 h+1\right)$ or $-4(h+1)\left(3 h^{2}+3 h+1\right)$. Then $E(\mathbb{Q})$ has no point of order 3.

Proof. As we mentioned in the proof of the previous lemma, the point $P=(x, y)$ is of order 3 if and only if $x$ is the root of

$$
\begin{aligned}
& T_{E}(X) \\
& =3(X+1)\left(X^{3}-X^{2}-(12 k+5) X-\left(12 k^{2}+12 k+3\right)\right) .
\end{aligned}
$$

Let $S_{E}(X)$ be the polynomial

$$
\begin{aligned}
& T_{E}(X) / 3(X+1) \\
& =X^{3}-X^{2}-(12 k+5) X-\left(12 k^{2}+12 k+3\right) .
\end{aligned}
$$

Since $\left(-1, \pm \sqrt{-3 k^{2}}\right)$ is not in $E(\mathbb{Q})$, it suffices to check whether $x$ is a root of $S_{E}(X)=0$ or not.

Suppose that $S_{E}(X)=0$ has a root $x^{\prime}$ in $\mathbb{Q}$. Then it is an integer. In other words, for an integer $k$ not the form $4 h\left(3 h^{2}+3 h+1\right)$ or $-4(h+1)\left(3 h^{2}+3 h+1\right)$ by sorting again as $k$, we can fine an integer $x^{\prime}$ such that

$$
\begin{aligned}
& x^{\prime 3}-x^{\prime 2}-(12 k+5) x^{\prime}-\left(12 k^{2}+12 k+3\right) \\
& =-12 k^{2}-12\left(x^{\prime}+1\right) k+x^{\prime 3}-x^{\prime 2}-5 x^{\prime}-3=0 .
\end{aligned}
$$


From, this $x^{\prime 3}-x^{\prime 2}-5 x^{\prime}-3$ must be a multiple of 12 and $x$ is one of $12 m+3,5,9$ or 11 for a suitable integer $m$.

When $x=12 m+3, S_{E}$ becomes $-12\left(k^{2}+12 k m+4 k-144 m^{3}-96 m^{2}-16 m\right)$. Because it has integral solutions as a quadratic equation with respect to $k$, its discriminant $16(4 m+1)(1+3 m)^{2}$ is a square. That means that $4 m+1=(2 h+1)^{2}$ for an integer $h$. Through this we get $k=4 h\left(3 h^{2}+3 h+1\right)$ or $-4(h+1)\left(3 h^{2}+3 h+1\right)$.

If $x=12 m+5,12 m+9$ or $x=12 m+11$ then discriminant of the quadratic equations with respect to $k$ is $3(12 m+5)\{2(2 m+1)\}_{2}^{2},(4 m+3)\{2(6 m+5)\}^{2}$ or $3(12 m+11)\{4(m+1)\}^{2}$ respectively. Neither case has a perfect square discriminant and admit any integral root. $\square$

Proof of Theorem 2. Use the Lemma 5 and Theorem 3 $3)$, we can determine which finite abelian group has a subgroup of $E(\mathbb{Q})$ for the case $k \equiv 1(\bmod 35)$, i.e., $k \equiv 1(\bmod 5)$ and $k \equiv 1(\bmod 7)$. In fact, $E(\mathbb{Q})_{\text {tors }}$ is a subgroup of both $\mathbb{Z} / 9 \mathbb{Z}$ and $\mathbb{Z} / 6 \mathbb{Z}$. It yields that it is $\mathbb{Z} / 3 \mathbb{Z}$ or trivial. Since our group has no point of order 3 , it is trivial.

Note that $E(\mathbb{Q})_{\text {tors }}$ is a subgroup of order $N$, if it is a subgroup of order $3^{r} \cdot N$ with $(3, N)=1$, then. So it is resolved as trivial group in many cases.

To observe easily, we can refer Table 2: In this table, $k$ takes the value modulo 5 at the horizontal line and modulo 7 at the vertical line respectively. The groups $C_{n}=\mathbb{Z} / n \mathbb{Z}$ in the brackets at top line and at the very left line are result from Lemma 5 .

Each entry implies that the type of group: "A", "B" or " $C$ " implies one of subgroups of $\mathbb{Z} / 4 \mathbb{Z}, \mathbb{Z} / 2 \mathbb{Z}$ or trivial, respectively. The same alphabet does not mean the same group. And "D" means that both curves $E_{5}\left(\mathbb{F}_{5}\right)$ and $E_{7}\left(\mathbb{F}_{7}\right)$ are singular. In this table since " $\mathrm{C}$ " is trivial, it remains that a few cases

$k \equiv 4,7,12,15,20,22,25,27,29,32$ or $34(\bmod 35)$.

For the cases that the subgroup is nontrivial Pro-

Table 2. Type of group $E(\mathbb{Q})_{\text {tors }}$.

\begin{tabular}{ccccccc}
\hline$k(\bmod 7)$ & $k(\bmod 5)$ & 0 & $1\left(C_{9}\right)$ & $2\left(C_{6}\right)$ & $3\left(C_{3}\right)$ & 4 \\
\hline 0 & & $\mathrm{D}$ & $\mathrm{C}$ & $\mathrm{B}$ & $\mathrm{C}$ & $\mathrm{D}$ \\
1 & $\left(C_{6}\right)$ & $\mathrm{B}$ & $\mathrm{C}$ & $\mathrm{B}$ & $\mathrm{C}$ & $\mathrm{B}$ \\
2 & $\left(C_{9}\right)$ & $\mathrm{C}$ & $\mathrm{C}$ & $\mathrm{C}$ & $\mathrm{C}$ & $\mathrm{C}$ \\
3 & $\left(C_{3} \oplus C_{3}\right)$ & $\mathrm{C}$ & $\mathrm{C}$ & $\mathrm{C}$ & $\mathrm{C}$ & $\mathrm{C}$ \\
4 & $\left(C_{6}\right)$ & $\mathrm{B}$ & $\mathrm{C}$ & $\mathrm{B}$ & $\mathrm{C}$ & $\mathrm{B}$ \\
5 & & $\mathrm{D}$ & $\mathrm{C}$ & $\mathrm{B}$ & $\mathrm{C}$ & $\mathrm{D}$ \\
6 & $\left(C_{12}\right)$ & $\mathrm{A}$ & $\mathrm{C}$ & $\mathrm{B}$ & $\mathrm{C}$ & $\mathrm{A}$ \\
\hline
\end{tabular}

position 6 makes us know which curve has the point of order 2 or not. Hence, it is sufficient to check the value $k$ having order 4 points.

Assume that $k \equiv 20,34(\bmod 35)$ and there exists an integer $l$ such that $k=-3 l(1-l)$. In fact $k \equiv 20(\bmod 35) \quad($ respectively, $34(\bmod 35))$ if and only if $l \equiv 5$ or $26(\bmod 35)$ (respectively, 19 or $33(\bmod 35)) .\left(3 l^{2}-1,0\right)$ is the unique point of order 2 . Using duplication formula for the elliptic curve, let $P=\left(x^{\prime}, y^{\prime}\right)$ be the point satisfying $2 P=\left(3 l^{2}-1,0\right)$. By Substituting $x^{\prime}, y^{\prime},-(6 k+3)$ and $-\left(3 k^{2}+6 k+2\right)$ for $x, y, A$ and $B$ in (in the formulas for $x_{2}$ and $y_{2}$ in the proof of Lemma 4), we get two equations affirming the existence of point of order 4:

$$
\begin{aligned}
& \left(x^{\prime 2}+2\left(1-3 l^{2}\right) x^{\prime}-18 l^{4}+18 l^{3}-6 l^{2}+1\right)^{2}=0 \\
& \left(x^{\prime 2}+2\left(1-3 l^{2}\right) x^{\prime}-18 l^{4}+18 l^{3}-6 l^{2}+1\right) \times F\left(x^{\prime}\right)=0
\end{aligned}
$$

where

$$
\begin{aligned}
F(x)= & x^{4}-2\left(1-3 l^{2}\right) x^{3}+6\left(9 l^{4}-18 l^{3}+12 l^{2}-2\right) x^{2} \\
& -2\left(54 l^{6}-162 l^{5}+108 l^{4}+54 l^{3}-63 l^{2}+7\right) x \\
& +324 l^{8}-972 l^{7}+864 l^{6}-270 l^{4}+60 l^{2}-5 .
\end{aligned}
$$

To have an integral solution of

$x^{2}+2\left(1-3 l^{2}\right) x-18 l^{4}+18 l^{3}-6 l^{2}+1=0$, its discriminant $36 l^{3}(3 l-2)$ have to be a square. Suppose that we can find an integer $m$ such that $m^{2}=l(3 l-2)$ and $x^{\prime}=3 l^{2}-1+6 l m$ ( or $3 l^{2}-1-6 l m$ ). It is easy to check that the integer $m$ satisfying the above condition exists in each case determined by $l$. Furthermore, by substituting $x^{\prime}, \quad k=-3 l(1-l)$ and $m^{2}=l(3 l-2)$ to the right hand side of (1.4) we get a numerical formula

$$
\begin{aligned}
& 54 l^{3}(3 l-2)\left(6 l^{2}-5 l m-2\right) \\
& =9 l^{2} \cdot 6 l(3 l-2) \cdot 6\left(6 l^{2}-5 l m-2\right) \\
& =9 l^{2} m^{2} \cdot 6\left(6 l^{2}-5 l m-2\right)
\end{aligned}
$$

Since $l \neq 0$ makes the curve (1.4) singular, $6\left(6 l^{2}-5 l m-2\right)$ is a square of a suitable integer if and only if there exists a point of order 4 .

So we are done. $\square$

\section{Conclusions}

By the help of Theorem 2, we explicitly calculate the torsion part of Modell-Weil group.

Example 8. Let $E: y^{2}=x^{3}-75 x-506$ be the elliptic curve. Then

$$
E(\mathbb{Q})_{\text {tors }}=\mathbb{Z} / 2 \mathbb{Z}
$$

Given elliptic curve is the form $k=12$ in Theorem 2 and $12=-3 \times 2^{2} \times(1-2)$. Therefore $E(\mathbb{Q})_{\text {tors }}=\mathbb{Z} / 2 \mathbb{Z}$. And $(11,0)$ is the nontrivial torsion point on $E(\mathbb{Q})$.

The method to find $E(\mathbb{Q})_{\text {tors }}$ is able to be applied to 
all elliptic curve without a condition for $k$ by choosing another prime $p>7$.

For example, in Theorem 2, there is a condition $35 \nmid k(9 k+4)$ for $k$. This is one for nonsingular curve. For the case that $35 \mid k(9 k+4)$, choose the another prime $p>7$ such that $p \nmid k(9 k+4)$. Calculate $E_{p}\left(\mathbb{F}_{p}\right)$ and eliminate the order 3 point and check the condition for having order 2 point. Since

$\left|E\left(\mathbb{F}_{p}\right)\right| \leq 2 p+1$, the smaller $p$ gives simpler necessary condition. For example, if $k=-16$ then the elliptic curve is

$$
E: y^{2}=x^{3}+93 x-674
$$

with discriminant $2^{6} \times 5 \times 7$. Find $E_{p}\left(\mathbb{Z}_{p}\right)$ with $p=11$ and $17,\left|E_{11}\left(\mathbb{Z}_{11}\right)\right|=15$ and $\left|E_{17}^{p}\left(\mathbb{Z}_{17}\right)\right|=18$. Using Lemma 4 , we observe that $E(\mathbb{Q})$ has no point of order 3 . So it is a trivial group.

Remark 9. Generalize our elliptic curve

$$
E: y^{2}=x^{3}+f(k) x+g(k)
$$

for $k \in \mathbb{Z}$ and $\max \{\operatorname{deg} f(k), \operatorname{deg} g(k)\} \leq 2$. We can use the criterion for the quadratic equation to find a point of order 2 or 3 . Of course, it is indispensable to consider some exceptional cases in the similar way to Proposition 7.

\section{REFERENCES}

[1] B. Mazur, "Modular Curves and the Eisenstein Ideal," Publications Mathématiques de l'Institut des Hautes Études Scientifiques, No. 47, 1977, pp. 33-168.

[2] A. Knapp, "Elliptic Curves," Princeton University Press, Princeton, 1992.

[3] D. Kim, J. K. Koo and Y. K. Park, "On the Elliptic Curves Modulo p," Journal of Number Theory, Vol. 128, No. 4, 2008, pp. 945-953. doi:10.1016/i.jnt.2007.04.015 